\title{
Antimicrobial resistance and epidemiology of ESBLs-producing Escherichia coli and Enterobacter cloacae isolates from the intensive care unit in an affiliated hospital of University, China
}

\section{Kun Chen}

Army Medical University

Guoliang Yang

Army Medical University

Wenping Li

Army Medical University

Mingcheng Li ( $\sim$ limingcheng@beihua.edu.cn)

Beihua University

Research

Keywords: Enterobacteriaceae, outbreak, ESBLs, infection control

Posted Date: June 26th, 2020

DOI: https://doi.org/10.21203/rs.3.rs-37906/v1

License: (c) (1) This work is licensed under a Creative Commons Attribution 4.0 International License.

Read Full License 


\section{Abstract}

Background冈Concerns are increasing over the importance of the hospital intensive care units (ICU) for the transmission of extended spectrum- $\beta$-lactamase (ESBLs) -producing Enterobacteriaceae. We reported the clinical characteristics and epidemiology of ESBLs isolates collected from a tertiary care hospital in China.

Methods $₫$ Escherichia coli E. coliand Enterobacter cloacae $\llbracket E$. cloacae『isolates from ICU infection samples were isolated and identified. Antimicrobial susceptibility profiles and production of ESBLs were determined by using the disk diffusion method and the broth microdilution method. Clonality of isolates was determined by ERIC-PCR techniques.

Results $\otimes$ From the included the 223 strains isolated from hospitalized patients with nosocomial infections in ICU during 2016 to 2018, the majority of isolates belonged to Gram-negative Enerobacteriaceae including $E$. coli ( $46.6 \%$ of all strains), and E. cloacae ( $46.2 \%$ of all strains). $63.25 \%$ of samples were separated from sputum or tracheal secretions. All of 207 isolates, ESBL-screen positive E. coli was 45.2\% (47/104), and 44.7\% (46/103) for E. cloacae. Resistance rates of ESBLs-producing $E$. coli and $E$. cloacae isolates were $95.5 \%-91.3 \%$ for ampicillin, $80.6 \%-76.1 \%$ for ampicillin/azobactam, $88.1 \%-28.3 \%$ for ciprofloxacin, $89.6 \%-15.2 \%$ for levofloxacin, $34.3 \%-45.7 \%$ for netilmicin, $82.1 \%-41.3 \%$ for compound sulfamethoxazole, $20.9 \%-43.5 \%$ for amikacin, $58.2 \%-37.0 \%$ for gentamicin, $20.9 \%-69.6 \%$ for piperacillin/tazobactam. All of ESBLs-producer isolates resistant to cefazolin, cefuroxime, ceftazidime, ceftriaxone, cefepime in additon to aztreonam were $100 \%$, whereas the susceptibilities of isolates to imipenem and meropenem were $100 \%$.

Results of ERIC-PCR in all of ESBLs-producing E. coli isolates exhibited 11 distinct patterns using a similarity coefficient of 0.8 . And one distinct ERIC profiles were observed amongst 46 strains of ESBLsproducing E. cloacae. ERIC profiles demonstrated an outbreak of nosocomial infection and ESBLsproducing E. coli and E. cloacae prevalent in the ICU of this hospital.

Conclusions®Our data indicate that the ESBLs-producing E. coli and E. cloacae clones are circulating in the ICU and constitute a major source for further disseminating in this hospital. It is necessary to increase surveillance and development of adequate prevention strategies.

\section{Introduction}

The incidence of infections due to the emergence and dissemination of Enerobacteriaceae has rapidly increased for the last several decades. Resistance of Enerobacteriaceae to extended-spectrum $\beta$-lactams worldwide is becoming a major public health problem[1]. Both Escherichia coli (E. coli) and Enterobacter cloacae (E. cloacae) presently are the ESBL producing Gram-negative Enerobacteriaceae, which have emerged as the most common cause of hospital-and community-acquired infection admitted to neonatal intensive-care settings [2]. The nosocomial infections of the intensive care units (ICU) that occur in the specific environment in which the treatment and rehabilitation of critically ill patients is frequently 
affected [3]. Known risk-factors for onset of infection in ICU with drug-resistant bacteria include: widespread abuse or irrational use of antibiotics, the rapid renewal of antibiotics, pathogens, in the specific ICU environment, critically illness, undergoing surgery, use of invasive medical devices, prolonged hospital stay, etc $[4,5]$. Onset of sepsis in ICU is the most common cause of infection-related mortality. Since appropriate antimicrobial treatment is essential for infections in ICU, institutional surveillance of infection-derived bacteria isolates and analysis of their susceptibility to different antimicrobial agents provides crucial information for the choice of empirical antimicrobial therapy. Besides that, the comprehensive analysis of ICU infection epidemiology and the evaluation of the prognosis of the disease are of great significance $[6,7]$.

The purpose of the current study mainly focused on the bacterial distribution and drug resistance characteristics of ICU infections. Furthermore, we aimed to explore the epidemiology of pathogenic bacteria transmission and diffusion resistance, through retrospective investigation on infectious cases in the ICU of a third-level hospital in Jilin from 2016 to 2018.

\section{Materials And Methods}

\section{Settings and collection of specimens}

This study was conducted at an affiliated hospital of University, one of the largest hospitals in Jilin of the North-East China, with approximately 2, 000 beds. We carried out a retrospective study of Enerobacteriaceaeinfected patients in the three ICU settings. 283 patients were enrolled in ICU from June 2016 to June 2018. The specimens were mainly obtained from sputum or tracheal secretions $(n=179)$, pus $\square n=33 \rrbracket$, blood $\square n=43 \rrbracket$, ascites $\square n=20 \rrbracket$, catheters and drainage tubes $\square n=7 \square$ and cerebrospinal fluid $\square n=2 \rrbracket$. No repetitive isolates from a single patient were included. This study to collect the clinical samples was approved by ethical committees of Beihua University (formal ethical approval number: Protocol Number 2016-01-01), and written informed consent was gained from all participants in the study prior to the initiation of the study.

\section{Identification and detection of resistance to 17 antibiotics agents}

The identification of E. coli and E. cloacae was identified by using the Vitek 2 Compact System with GN card and ASTGN13 card (bioMérieux, Marcy l'Etoile, France). Susceptibility to a panel of 16 antimicrobial agents was determined according to Clinical and Laboratory Standards Institute recommendations (CLSI, 2012) [8]. The tested antibiotics (AB Biodisk, Solna, Sweden) were included: ampicillin, cefazolin, cefuroxime, ceftazidime, ceftriaxone, cefepime, levofloxacin, netilmicin, aztreonam, ciprofloxacin, amikacin, gentamicin, imipenem, meropenem, ampicillin /azobactam, piperacillin / tazobactam and compound sulfamethoxazole.

MIC values for 17 drugs at 12 concentrations in vitro were determined using a micro broth dilution method. E. coli ATTCC 25922, K. pneumoniae 700603 (ESBL positive) and E. cloacae ATCC 13047 were 
used as quality control strains.

Determination of ESBLs-producing strains

Suspected ESBLs-producing strains were screened using cefotaxime $(30 \mu \mathrm{g})$ and ceftazidime $(30 \mu \mathrm{g})$ in combination with clavulanic acid $(10 \mu \mathrm{g})$ according to the CLSI recommended disk diffusion method [8].

Clonality analysis of E. coli and E. cloacae isolates by ERIC

\section{Total DNA extraction from E. coli and E. cloacae}

A single colony was selected from the passage medium and incubated overnight at $37^{\circ} \mathrm{C}$ after being added to a test tube containing $2 \mathrm{ml}$ of Luria-Bertani liquid. The next day, $2 \mathrm{ml}$ of bacterial liquid was centrifuged at 12, $000 \mathrm{r} / \mathrm{min}$ for 5 minutes. The supernatant was discarded and added to $400 \mu \mathrm{ldd} \mathrm{H}_{2} \mathrm{O}$ and boiled for 10 minutes after mixing. The mixture was cooled and centrifuged at $12,000 \mathrm{r} / \mathrm{min}$ for 5 minutes, and the supernatant was absorbed and stored at $-20^{\circ} \mathrm{C}$.

\section{ERIC-PCR amplification}

The primer sequences were P1-ATGTAAGCTCCTGGGGATTCAC and P2- AAGTAAGTGACTGGGGTGAGCG. The system contained $2 \mu \mathrm{l}$ of $\mathrm{P} 1$ and $\mathrm{P} 2$ primers, and the DNA template solution at $3 \mu \mathrm{g} / \mathrm{l}$. $\mathrm{ddH}_{2} \mathrm{O}$ was added to the total reaction system of $50 \mu$ l. PCRs were conducted in a GeneAmp PCR system 9600 (Perkin-Elmer, USA) under the following reaction conditions; denaturation at $94{ }^{\circ} \mathrm{C}$ for 5 min, denaturation at $94{ }^{\circ} \mathrm{C}$ for $30 \mathrm{~s}$, annealing at $56 \otimes 58^{\circ} \mathrm{C}$ for $45 \mathrm{~s}$, extension at $72{ }^{\circ} \mathrm{C}$ for $30 \mathrm{~s}$, and 32 cycles at $72{ }^{\circ} \mathrm{C}$ after $5 \mathrm{~min}$. PCR products were analyzed by $2 \%$ agarose gel electrophoresis at $60 \mathrm{v}$ for $40 \mathrm{~min}$. A molecular weight DNA Marker from 100冈600 bp was used for reference and a gel imaging analysis system was used to observe and analyze the results. Band comparisons were carried out by clustering analysis with the unweighted pair group method using Quantity One (Version 4.6.2). Isolates were considered as the same origins if their similarity coefficients were equal to or more over 0.8 , whereas, lower 0.8 is different origins according to the reference [9].

\section{Statistical analysis}

Differences in the drug resistance rates of non-ESBLs-producing and ESBLs-producing strains were tested by Chi-square test. All drug resistant data were analyzed using SPSS13.0 statistical software. A value of $P<0.05$ was considered as statistically significant.

\section{Results}

\section{Epidemiological characteristics of specimens and isolates}

From the included the 223 strains isolated from hospitalized patients with nosocomial infections in ICU during 2016 to 2018, the majority of isolates belonged to Gram-negative Enerobacteriaceae including $E$. coli (46.6\% of all strains), which accounted for 24 strains collected in 2016, 55 strains in 2017 and 25 
strains in 2018; E. cloacae (46.2\% of all strains), which accounted for 20 strains collected in 2016, 61 strains in 2017 and 22 strains in 2018; others were not included in the study (data not shown).

The clinical distribution of the specimens was mainly composed of sputum or tracheal secretions accounting for $63.3 \%$ of the samples, followed by skin and purulent infections(11.5\%), blood $15.2 \% \bigotimes$, ascites $₫ 7.1 \% \bigotimes$, catheters and drainage tubes $\varangle 2.4 \% \bigotimes$ and cerebrospinal fluid $\varangle 0.5 \% \bigotimes$.

\section{Determination of ESBLs phenotypes}

In the primary screening test, 72 strains of $E$. coli in the inhibitory zone to ceftazidime with a diameter of $\leq 22 \mathrm{~mm}$, and the inhibitory zone to ceftriaxone, which was $\leq 25 \mathrm{~mm}$ in diameter, were highly suspected ESBLs-producing bacteria. It was confirmed that 67 strains of $E$. coli alone were detected in the inhibitory zone to cephalosporin which had a diameter of $\geq 5 \mathrm{~mm}$. At the same time, the diameter of the inhibitory zone to clavulanic acid was $\geq 5 \mathrm{~mm}$.

All of 103 strains of E. cloacae, 61 strains of E. cloacae to ceftazidime and ceftriaxone were highly suspected to produce ESBLs in the screening test. The inhibitory zone to ceftazidime with a diameter of $\leq 22 \mathrm{~mm}$ and the inhibitory zone to ceftriaxone which was $\leq 25 \mathrm{~mm}$ in diameter were highly suspected to produce ESBLs. It was confirmed that there were 46 strains of E. cloacae isolates whose inhibitory zone diameter to cephalosporin was $\geq 5 \mathrm{~mm}$. Meanwhile, the diameter of the inhibitory zone to clavulanic acid was $\geq 5 \mathrm{~mm}$.

\section{Antimicrobial susceptibility of E. coli and E. cloacae}

Resistance rates of ESBLs-producing E. coli and E. cloacae isolates were 95.5\%-91.3\% for ampicillin, 80.6\%-76.1\% for ampicillin/azobactam, 88.1\%-28.3\% for ciprofloxacin, 89.6\%-15.2\% for levofloxacin, $34.3 \%-45.7 \%$ for netilmicin, $82.1 \%-41.3 \%$ for compound sulfamethoxazole, $20.9 \%-43.5 \%$ for amikacin, $58.2 \%-37.0 \%$ for gentamicin, $20.9 \%-69.6 \%$ for piperacillin/tazobactam. All of ESBLs-producer isolates resistant to cefazolin, cefuroxime, ceftazidime, ceftriaxone, cefepime in additon to aztreonam were $100 \%$, whereas the susceptibilities of isolates to imipenem, meropenem were $100 \%$.

The susceptibilities of non-ESBLs-screen positive E. coli and E. cloacae isolates were $89.2 \%-91.3 \%$ to ampicillin, $97.3 \%-96.5 \%$ to ampicillin/azobactam, $94.6 \%-94.7 \%$ to cefazolin, $89.2 \%-96.5 \%$ to cefuroxime, 97.3\%-98.3\% to ceftazidime, $97.3 \%-98.3 \%$ to ceftriaxone, $91.9 \%-92.3 \%$ to cefepime, $89.2 \%-100.0 \%$ to aztreonam,32.4\%-68.4\% to ciprofloxacin, $37.8 \%-91.2 \%$ to levofloxacin, $70.3 \%-56.1 \%$ to netilmicin, $43.2 \%-61.4 \%$ to compound sulfamethoxazole, $62.2 \%-71.9 \%$ to amikacin, $43.2 \%-68.4 \%$ to gentamicin, $97.3 \%-93.0 \%$ to piperacillin/tazobactam respectively (Table 1, Table 2 ). Only the susceptibilities to imipenem and meropenem were $100 \%$. 
Table 1

Susceptibility to common antimicrobials of 104 E. coli isolates

\begin{tabular}{|c|c|c|c|c|c|c|}
\hline \multirow[t]{2}{*}{ Antimicrobiol agents } & \multicolumn{2}{|c|}{$\begin{array}{l}\text { ESBL positive } \\
(n=67)\end{array}$} & \multicolumn{2}{|c|}{$\begin{array}{l}\text { ESBL negative } \\
(n=37)\end{array}$} & \multirow[t]{2}{*}{$x^{2}$} & \multirow[t]{2}{*}{$\begin{array}{l}P \\
\text { value }\end{array}$} \\
\hline & $\mathrm{R}(\%) *$ & $\mathrm{~S}(\%)$ & $\mathrm{R}(\%)$ & $\mathrm{S}(\%)$ & & \\
\hline Ampicillin & $64(95.5)$ & $3(4.5)$ & $4(10.8)$ & $33(89.2)$ & 4.42 & $\begin{array}{l}<.005 \\
0.005\end{array}$ \\
\hline Ampicillin /azobactam & $54(80.6)$ & 13(19.4) & $1(2.7)$ & $36(97.3)$ & 5.01 & $\begin{array}{l}< \\
0.005\end{array}$ \\
\hline Cefazolin & $67(100.0)$ & $0(0.0)$ & $2(5.4)$ & $35(94.6)$ & 130.67 & $<.005$ \\
\hline Cefuroxime & $67(100.0)$ & $0(0.0)$ & $4(10.8)$ & 33(89.2) & 169.60 & $<.005$ \\
\hline Ceftazidime & $67(100.0)$ & $0(0.0)$ & $1(2.7)$ & $36(97.3)$ & 24.34 & $\begin{array}{l}< \\
0.005\end{array}$ \\
\hline Ceftriaxone & $67(100.0)$ & $0(0.0)$ & $3(8.1)$ & $34(91.9)$ & 83.79 & $\begin{array}{l}< \\
0.005\end{array}$ \\
\hline Cefepime & $67(100.0)$ & $0(0.0)$ & $3(8.1)$ & $34(91.9)$ & 19.51 & $<.005$ \\
\hline Aztreonam & $67(100.0)$ & $0(0.0)$ & $4(10.8)$ & $33(89.2)$ & 88.48 & $<.005$ \\
\hline Ciprofloxacin & $59(88.1)$ & $8(11.9)$ & $25(67.6)$ & 12(32.4) & 3.31 & $\varangle 0.005$ \\
\hline Gentamicin & $39(58.2)$ & $28(41.8)$ & $21(56.8)$ & $16(43.2)$ & 3.80 & $\begin{array}{l}< \\
0.005\end{array}$ \\
\hline Imipenem & $0(0.0)$ & $67(100.0)$ & $0(0.0)$ & $37(100.0)$ & 2.01 & $\otimes 0.005$ \\
\hline Meropenem & $0(0.0)$ & $67(100.0)$ & $0(0.0)$ & $37(100.0)$ & 0 & 0 \\
\hline Levofloxacin & $60(89.6)$ & $7(10.5)$ & $23(62.2)$ & 14(37.8) & 4.69 & $<.005$ \\
\hline Netilmicin & $23(34.3)$ & $44(65.7)$ & $11(29.7)$ & $26(70.3)$ & 4.91 & $\begin{array}{l}< \\
0.005\end{array}$ \\
\hline $\begin{array}{l}\text { Compound } \\
\text { sulfamethoxazole }\end{array}$ & $55(82.1)$ & 12(17.9) & $21(56.8)$ & $16(43.2)$ & 61.68 & $<.005$ \\
\hline Piperacillin / tazobactam & 14(20.9) & $53(79.1)$ & $1(2.7)$ & $36(97.3)$ & 4.54 & $\begin{array}{l}< \\
0.005\end{array}$ \\
\hline Amikacin & $20(29.9)$ & $47(70.2)$ & 14(37.8) & $23(62.2)$ & 4.74 & $<.005$ \\
\hline
\end{tabular}


Note: ${ }^{*} P<0.05$ compared with the ESBL negative group 
Table 2

Susceptibility to common antimicrobials of 103 E. cloacae isolates

\begin{tabular}{|c|c|c|c|c|c|c|}
\hline \multirow[t]{2}{*}{ Antimicrobiol agents } & \multicolumn{2}{|c|}{$\begin{array}{l}\text { ESBL positive } \\
(n=46)\end{array}$} & \multicolumn{2}{|c|}{$\begin{array}{l}\text { ESBL negative } \\
(n=57)\end{array}$} & \multirow[t]{2}{*}{$x^{2}$} & \multirow[t]{2}{*}{$\begin{array}{l}P \\
\text { value }\end{array}$} \\
\hline & $\mathrm{R}(\%) *$ & $S(\%)$ & $\mathrm{R}(\%)$ & $S(\%)$ & & \\
\hline Ampicillin & $42(91.3)$ & $4(8.7)$ & $4(7.0)$ & $53(93.0)$ & 4.42 & $<.005$ \\
\hline Ampicillin /azobactam & $35(76.1)$ & 11(23.9) & $2(3.5)$ & $55(96.5)$ & 4.52 & ${ }_{0.005}^{<}$ \\
\hline Cefazolin & $46(100.0)$ & $0(0.0)$ & $3(5.3)$ & $54(94.7)$ & 130.67 & $\begin{array}{l}< \\
0.005\end{array}$ \\
\hline Cefuroxime & $46(100.0)$ & $0(0.0)$ & $2(3.5)$ & $55(96.5)$ & 169.60 & $<005$ \\
\hline Ceftazidime & $46(100.0)$ & $0(0.0)$ & $1(1.8)$ & $56(98.3)$ & 24.34 & $\begin{array}{l}< \\
0.005\end{array}$ \\
\hline Ceftriaxone & $46(100.0)$ & $0(0.0)$ & $4(7.0)$ & $53(93.0)$ & 83.79 & $\begin{array}{l}< \\
0.005\end{array}$ \\
\hline Cefepime & $46(100.0)$ & $0(0.0)$ & $2(3.5)$ & $55(96.5)$ & 19.51 & $\begin{array}{l}< \\
0.005\end{array}$ \\
\hline Aztreonam & $46(100.0)$ & $0(0.0)$ & $0(0.0)$ & $57(100.0)$ & 88.48 & $\begin{array}{l}<.005 \\
0.00\end{array}$ \\
\hline Ciprofloxacin & 13(28.3) & $33(71.7)$ & 18(31.6) & $39(68.4)$ & 3.31 & $\varangle 0.005$ \\
\hline Gentamicin & 17(37) & $29(63.0)$ & $20(35.1)$ & $37(64.9)$ & 3.80 & $\begin{array}{l}< \\
0.005\end{array}$ \\
\hline Imipenem & $0(0.0)$ & $46(100.0)$ & $0(0.0)$ & $57(100.0)$ & 2.01 & $\varangle 0.005$ \\
\hline Meropenem & $0(0.0)$ & $46(100.0)$ & $0(0.0)$ & $57(100.0)$ & 0 & 0 \\
\hline Levofloxacin & $7(15.2)$ & $39(84.8)$ & $5(8.8)$ & $52(91.2)$ & 4.69 & $\begin{array}{l}<.005 \\
0.00\end{array}$ \\
\hline Netilmicin & $21(45.7)$ & $25(54.4)$ & $25(43.9)$ & $32(56.1)$ & 4.91 & $<005$ \\
\hline $\begin{array}{l}\text { Compound } \\
\text { sulfamethoxazole }\end{array}$ & 19(41.3) & $27(50.7)$ & $22(38.6)$ & $35(61.4)$ & 61.68 & $<0.005$ \\
\hline Piperacillin / tazobactam & $32(69.6)$ & 14(30.4) & $4(7.0)$ & $53(93.0)$ & 4.21 & $<005$ \\
\hline Amikacin & $20(43.5)$ & $26(56.5)$ & $16(28.1)$ & 41(71.9) & 4.27 & $<0.005$ \\
\hline
\end{tabular}


Note: ${ }^{*} P<0.05$ compared with the ESBL negative group

Next, we investigated a local difference of antibiotic resistance between ESBLs and non-EBSLs strains. There was significant difference between non-ESBLs-producing strains and ESBLs-producing strains $(P<$ $0.05)$ except the susceptibility to ciprofloxacin (Pख0.05).

\section{Determination of MIC values}

The MIC values of 67 strains of ESBLs-producing E. coli against ampicillin, ceftriaxone, cefazolin, aztreonam, cefixime and ceftazidime were more than $256 \mu \mathrm{g} / \mathrm{ml}$. The MIC for both levofloxacin and cotrimoxazole was greater than $128 \mu \mathrm{g} / \mathrm{ml}$. Ampicillin/sulbactam and piperacillin/tazobactam were highly resistant to ampicillin / sulbactam (MIC $=32 \mu \mathrm{g} / \mathrm{ml})$.

The MIC of 46 strains of ESBLs-producing E. cloacae against ampicillin, ceftriaxone, cefazolin, aztreonam, cefixime and ceftazidime was all greater than $256 \mu \mathrm{g} / \mathrm{ml}$. The MIC of amikacin, netilmicin, ampicillin/sulbactam and piperacillin/tazobactam was $32 \mu \mathrm{g} / \mathrm{ml}$.

\section{Clonality of isolates analysis by ERIC-PCR}

Clonality of isolates analysis of all the ESBLs-producing strains was investigated by ERIC-PCR typing. Based on the ERIC-PCR typing, one distinct ERIC profiles were observed amongst 46 strains of ESBLsproducing E. cloacae, showing that these isolates were of the equal clones (Fig. 1). We selected a representative of the same band in $67 \mathrm{ESBLs}$-producing $E$. coli isolates from different samples for dendrogram cluster analysis, revealed 11 distinct patterns using a similarity coefficient of 0.8 . An obvious clonal association was found within these strains, of which $54(80.6 \%)$ were of the identical clones (Figs. 2 and 3), indicating an outbreak situation. The history of the same drug-resistant strains in these ICU patients was similar. Most of the drug-resistant strains with the same clones had more infections in the lower respiratory tract, and $76 \%$ of patients had a history of mechanical ventilation.

\section{Discussion}

Antibacterial drugs have been administered extensively in clinic, especially in the treatment of ICU patients. However, the widespread use of antibacterial drugs produces further resistant bacteria, which is becoming a serious public health concern [10]. The abuse of this antibacterial drug causes the body to produce multi-drug resistance and the risk of super bacterial infection increases. The rational application of antibacterial drugs in the clinic effectively prevents the spread of drug-resistant strains and more comprehensive understanding of drug resistance mechanisms, and epidemiology of drug-resistant strains has much potential to improve the cure rates of ICU patients [11].

The majority of ICU isolates collected in the participating multicenters consisted of E. coli and Klebsiella pneumoniae in additon to E. cloacae in China $[12,13]$. In this study, ESBLs-producing and non-ESBLsproducing strains of $E$. coli showed significant differences in resistance to cephalosporins with ESBLsproducing strains having significantly higher resistance rates. In contrast, there was no obvious 
difference between the ESBLs-producing and non-ESBLs-producing strains concerning ciprofloxacin. In the comparison to the sensitivity of cephalosporin antibiotics with sulbactam and tazobactam, the drug resistance was extremely lower than that of cephalosporins alone. This group of studies fully demonstrated that if the production of ESBLs can be reduced as much as possible, the resistance of $E$. coli can also be greatly reduced.

E. coli and E. cloacae belong to conditional pathogens that are part of the normal intestinal flora but can cause infections of the respiratory and urinary tracts $[14,15]$. An epidemiological study has reported that the detection rate of $E$. coli and E. cloacae producing ESBLs was increasing, $44 \%$ in Singapore, $37 \%$ in China [16]. Both E. coli and E. cloacae producing ESBLs are closely related to the drug resistance of antibiotics, and the same type of bacteria are likely to carry a variety of ESBLs, leading to multi-drug resistance $[17,18]$. Studies have also shown that there are multiple drug resistance mechanisms in $E$. coli and E. cloacae, which include mutations in Amp C enzymes and porin loss, which has been described in previous literatures $[19,20]$. However, we did not explore molucular mechanisms of resistances.

In this study, we collected samples from different parts of the patients and found that $E$. coli and $E$. cloacae were most common in infections of the respiratory tract and skin. The resistance of E. cloacae to 17 antimicrobial agents was similar to that of E. coli. All of them exhibited high resistance to cephalosporins, whilst they were highly sensitive to carbapenems. Because the northeast part of China is the most colded region with the highest incurrence of respiratory system diseases, antimicrobial overuse might be an explanation for the antimicrobial agents susceptibility difference in this area.

Clinically, the application of a large number of high-grade cephalosporin antibiotics in the treatment of ICU patients with nosocomial infections and unreasonable use of the third-generation cephalosporins have resulted in a large number of drug-resistant strains [21, 22]. Patients who are ineffectively treated with advanced cephalosporins or $\beta$-lactamase inhibitors can only further use carbapenem antibacterial drugs in treating Gram-negative bacteria [23].

Carbapenems have the strongest antimicrobial activity, broadest antimicrobial spectrum and belong to atypical beta-lactam antimicrobial agents. The low toxicity and high stability of beta-lactamase make them one of the best options for the treatment of severe infections when other antimicrobial agents are ineffective [24, 25].

In this study, both E. coli and E. cloacae were highly sensitive to carbapenems, and no significant difference in resistance between ESBLs-producing and non-ESBLs-producing strains was observed, indicating that carbapenems could be used as an option in the treatment of ESBLs-resistant strains. In contrast to data collected from centers in east China, the reduced susceptibilities of E. coli and E. cloacae indicated a local carbapenem resistance [26]. Taken together, the carbapenem overuse might be an explanantion for the difference because of the developed areas with the higher incomes [27].

In the epidemiological analysis of nosocomial infections in the ICU, ERIC-PCR was used to identify isolates EIC-PCR fingerprints. Results of ERIC-PCR in all of ESBLs-producing E. coli isolates exhibited 11 
distinct profiles, and one distinct ERIC profiles were observed amongst 46 strains of ESBLs-producing $E$. cloacae. Since the establishment of ERIC-PCR technique by versalovic et al. in 1991, it has played an important role in the identification and epidemiological investigation of Gram-negative bacteria. In 2001, Matsumoto et al. applied two different methods, ERIC-PCR and PFGE, to detect 23 clinical isolates of Berkholderia onions. The results indicated that two methods have similar detection abilities and reproducibility $[28,29]$. ERIC profiles demonstrated an outbreak of nosocomial infection and ESBLsproducing E. coli and E. cloacae prevalent in the ICU of this hospital.

The study found that ESBLs-producing bacteria were resistant to aminoglycoside antibiotics such as levofloxacin, and were more resistant to the above two types of antibacterial drugs than those with high resistance to cephalosporin antibiotics. The resistance to synthetic antibiotics such as sulfonamides was also lower than that of simple ampicillin. In the clinical application of antibiotics, the initial use of advanced cephalosporin antibiotics, especially the unreasonable use of the third generation cephalosporins, is the beginning of habitual therapy. These practices cause us to ignore the role of aminoglycosides and other antibacterial drugs that lead to common clinical drug resistance. In order to control the production and spread of ESBLs from the source, more reasonable use of antibacterial drugs should be followed by limiting the routine empirical application of high-level antibiotics by medical personnel. Publicity and education on the use of antibacterial drugs amongst health care workers should be strengthened along with improvements in the management of pharmacies and hospital ICUs [30, 31]. These measures could effectively cut off the source of infection and a mode of transmission of ESBLs bacteria. In addition, this could guide medical staff to rationally use antimicrobial agents [32]. This study has some limitations. Firstly, the genotypic or molecular data of all strains were not documented.

Secondly, the molecular epidemiology was not included. Further studies are needed to confirm the genetic types and the mechanism of transmission.

\section{Conclusions}

our findings indicate that the ESBLs-producing E. coli and E. cloacae clones are circulating in the ICU and constitute a major source for further disseminating in this hospital. Carbapenems are the reasonable choice in the treatment of ESBLs-producing bacteria. It is necessary to increase surveillance and development of adequate prevention strategies.

\section{Abbreviations}

ICU: intensive care units; ESBLs:extended spectrum- $\beta$-lactamase; E. cloacae:Enterobacter cloacae;E. coli:Escherichia coli; ERIC:Enterobacterial repetitive intergenic consensus.

\section{Declarations}

\section{Acknowledgement}


Not applicable.

\section{Authors' contributions}

Ken Chen conceived, designed the experiments and wrote a draft manuscript. Mingcheng Li analyzed, interpreted the results of the experiments and revised the manuscript. Guoliang Yang and Wenping Li performed the experiments. All authors read and approved the final manuscript.

\section{Funding}

The present project was a part of National Natural Science Foundation of China project (81402979), the Jilin Science and Technology Development Program (20140307008YY and 2014C33155).

\section{Availability of data and materials}

All data generated or analyzed during this study are available in this published article.

\section{Ethics approval and consent to participate}

This study to collect the clinical samples was approved by ethical committees of Beihua University (formal ethical approval number: Protocol Number 2016-01-01), and written informed consent was gained from all participants in the study prior to the initiation of the study.

\section{Consent for publication}

Not applicable

\section{Competing interests}

The authors declare that they have no competing interests. The authors alone are responsible for the content and writing of the paper.

\section{Author details}

${ }^{1}$ Department of Clinical Nursing, School of Nursing, Army Medical University, Chongqing, 400042, China

${ }^{2}$ Department of Ultrasound, XinQiao Hospital, Army Medical University, Chongqing, 400042, China

${ }^{3}$ Department of Obstetrics and Gynecology, DaPing Hospital, Army Medical Univerity, Chongqing, 400042, China

${ }^{4}$ School of Laboratory Medicine, Beihua University, Jilin, 132013, China

\section{References}


1. Singh N, Yu VL. Rational empiric antibiotic prescricption in the ICU. Chest. 2000;117:1496-9.

2. Ripabelli G, Tamburro M, Guerrizio G, Fanelli I, Flocco R, Scutellà $M$, et al. Tracking multidrug-resistant Klebsiella Pneumoniae from an Italian Hospital: molecular epidemiology and surveillance by PFGE, RAPD and PCR-based resistance genes prevalence. Curr Microbiol. 2018;75(8):977-87.

3. Yoon EJ, Hong JS, Yang JW, Lee KJ, Lee H, Jeong SH. Detection of mcr-1 plasmids in Enterobacteriaceae isolates from human specimens: comparison with those in Escherichia coli isolates from livestock in Korea. Ann Lab Med. 2018;38(6):555-62.

4. Bradford PA. Extended-spactrum $\beta$-lactamases in the 21st century: characterization, epidemiology, and detection of this important resistance threat. Clin microbial rev. 2014;14(4):933-51.

5. Mulvey MR, Bryce E, Boyd D, Ofner-Agostini M, Christianson S, Simor AE, et al. Ambler class A extended-spectrum beta- lactamase-producing Escherichia coli and klebsiella spp. In Canadian hospital. Antimicrob Agents Chemother. 2004;48:1204-14.

6. Quinteros M, Radice M, Gardella N, Rodriguez MM, Costa N, Korbenfeld D, et al. Extended-spectrumbeta-lactamase in Enterobacteriaceae in Buenes Aires, Srgentiana, public hospitals. Antimicrob Agents Chemother. 2003;47:2864-7.

7. Ripabelli G, Sammarco ML, Scutellà M, Felice V, Tamburro M. Carbapenem-Resistant KPC-. and TEMProducing Escherichia coli ST131 Isolated from a Hospitalized Patient with Urinary Tract Infection: First Isolation in Molise Region, Central Italy, July 2018. Microb Drug Resist. doi.org/10.1089/mdr.2019.0085.

8. Clincal and Laboratory Standards Institute. Performance Standards for Antimicrobial Susceptibility Testings; Nineteeth Informational Supplement, M100-S19-Wayne, PA, USA. CLSI, 2012.

9. Su DH, Lu JC, Zuo C, Li HY, Chen DM, Wang M, et al. Resistance of ESBLs-producing Escherichia coli and Klebsiella pneumoniae isolates with CTX-M genotypes in Guangzhou: analysis of their genotyping. Chin J Nosocomiol. 2009;19(22):3004-6.

10. Tian SF, Chen BY, Chu YZ, Wang S. Prevalence of rectal carriage of extended-spectrum-betalactamase-producing Escherichia coli among elderly people in community setting in China. Can J Microbiol. 2008;54(9):781-5.

11. Rordorf G, KoroshetzW, Efird JT, Cramer SC. Predictors of mortality in stroke patients admitted to an intensive care unit. Crit Care Med. 2014;28:1301-5.

12. Zhang R, Ichijo T, Hu YY, Zhou HW, Yamaguchi N, Nasu M, et al. A ten years (2000-2009) surveillance of resistant Entero- bacteriaceae in Zhejiang Province, China. Microb Ecol Health Dis. 2012;23.

13. Vincent JL. Nosocomial infections in adult intensive-care units. Lancet. 2014;361:2068-77.

14. Gniadkowski M. Evolution and epidemiology of extended-spectrum beta-lactamases (ESBLs) and ESBLs-producing micoorganisms. Clin Microbiol Infect. 2014;7(11):597-608.

15. Nathisuwan S, Burgess DS, Lewis JS 2. Extended-spectrum beta-lactamases: epidemiology, detection and treatment. Pharmacotherapy. 2014;21(8):902-28. nd. ; ). 
16. Vinué L, Sáenz Y, Martínez S, Somalo S, Moreno MA, Torres $C$, et al. Prevalence and diversity of extended spectrum-beta -lactamases in faecal Escherichia coli isolates from healthy humans in Spain. Clin Microbiol Infect. 2009;15(10):954-7.

17. Magiorakos AP, Srinivasan A, Carey RB, Carmeli Y, Falagas ME, Giske CG,et al. Multidrug-resistant, extensively drug-resistant and pandrug-resistant bacteria: an international expert proposal for interim standard definitions for acquired resistance. Clin Microbiol Infect. 2012;18:268-81.

18. Leistner R, Schröder C, Geffers C, Breier AC, Gastmeier P, Behnke M. Regional distribution of nosocomial infections due to ESBL-positive Enterobacteriaceae in Germany: data from the German National Reference Center for the Surveillance of Nosocomial Infections (KISS). Clin Microbiol Infect. 2015;21(255):e1-5.

19. Zhang H, Yang Q, Liao K, Ni Y, Yu Y, Hu B, et al. Update of incidence and antimicrobial susceptibility trends of Escherichia coli and Klebsiella pneumoniae isolates from Chinese intra-abdominal infection patients. BMC Infect Dis. 2017;17:776-85.

20. Castanheira M, Farrell SE, Deshpande LM, Mendes RE, Jones RN. Prevalence of $\beta$-lactamase encoding genes among Enterobacteriaceae bacteremia isolates collected in $26 \mathrm{U}$. S. A. hospitals: report from the SENTRY Antimicrobial Surveillance Program (2010). Antimicrob Agents Chemother. 2013;57:3012-20.

21. Han SH, Kim YA, Wang M, Lee Y, Chung HS, Yum JH, et al. Comparison of the genetic structures surrounding qnrA1 in Korean Enterobactercloacae and Chinese Escherichiacoli strains isolated in the early 2000s: evidence for qnrA mobilization via Inc HI2 type plasmid. J Microbiol. 2012;50(1):166-9.

22. Qu H, Wang X, Ni Y, Liu J, Tan R, Huang J, et al. NDM-1-producing Enterobacteriaceae in a teaching hospital in Shanghai, China: IncX3-type plasmids may contribute to the dissemination of $b / a_{\mathrm{NDM}-1}$. Int J Infect Dis. 2015;34:8-13.

23. Carattoli A. Resistance plasmid families in Enterobacteriaceae. Antimicrob Agents Chemother. 2009;53(6):2227-38.

24. Yang $Q$, Zhang $H$, Wang $Y, X u Y$, Chen $M$, Badal RE, et al. A 10 year surveillance for antimicrobial susceptibility of Escherichia Coli and Klebsiella Pneumoniae in community- and hospital-associated intra-abdominal infections in China. J Med Microbiol. 2013;62:1343-9.

25. Du N, Liu S, Niu M, Duan Y, Zhang S, Yao J, et al. Transmission and characterization of bla $\mathrm{NDM}_{-1}$ in Enterobacter cloacae at a teaching hospital in Yunnan, China. Ann Clin Microbiol Antimicrob. 2017;16(1):58.

26. Charfi K, Grami R, Ben Jeddou A, Messaoudi A, Mani Y, Bouallegue O, et al. Extended-spectrum $\beta$ lactamases and plasmid-mediated quinolone resistance in enterobacterial clinical isolates from neonates in Tunisia. Microb Pathog. 2017;110:184-8.

27. Cohen J. Confronting the threat of multidrug-resistant Gram-negative bacteria in critically ill patients. J Antimicrob Chemother. 2013;68:490-1.

28. Chanawong A, M'Zali FH, Heritage J, Xiong JH, Hawkey PM. Three cefotaximases. CTX-M-9, CTX-M13, and CTX-M-14, among Enterobacteriaceae in the People's Republic of China. Antimicrob Agents 
Chemother. 2002;46:630-7.

29. Matsumoto M, Suzuki Y, Miyazaki Y, Tanaka D, Yasuoka T, Mashiko K, et al. Enterobacterial repetitive intergenic consensus sequence-based PCR (ERIC-PCR); its ability to differentiate Streptococcus pyogenes strains and applicability to the study of outbreak of streptocoacal infection. Tohoku J Exp Med. 2001;194:205-12.

30. Woodford N, Turton JF, Livermore DM. Multiresistant Gram-negative bacteria: The role of high-risk clones in the dissemination of antibiotic resistance. FEMS Microbiol Rev. 2011;35:736-55.

31. Luo Y, Yang J, Ye L, Guo L, Zhao Q, Chen R, et al. Characterization of KPC-2-producing Escherichia coli, Citrobacter freundii, Enterobacter cloacae, Enterobacter aerogenes, and Klebsiella oxytoca isolates from a Chinese Hospital. Microb Drug Resist. 2014; 20(4):264-9.

32. Hara T, Sato T, Horiyama T, Kanazawa S, Yamaguchi T, Maki H. Prevalence and molecular characterization of CTX-M extended-spectrum betalactamase-producing Escherichia coli from 2000 to 2010 in Japan. Jpn J Antibiot. 2015;68:75-84.

\section{Figures}

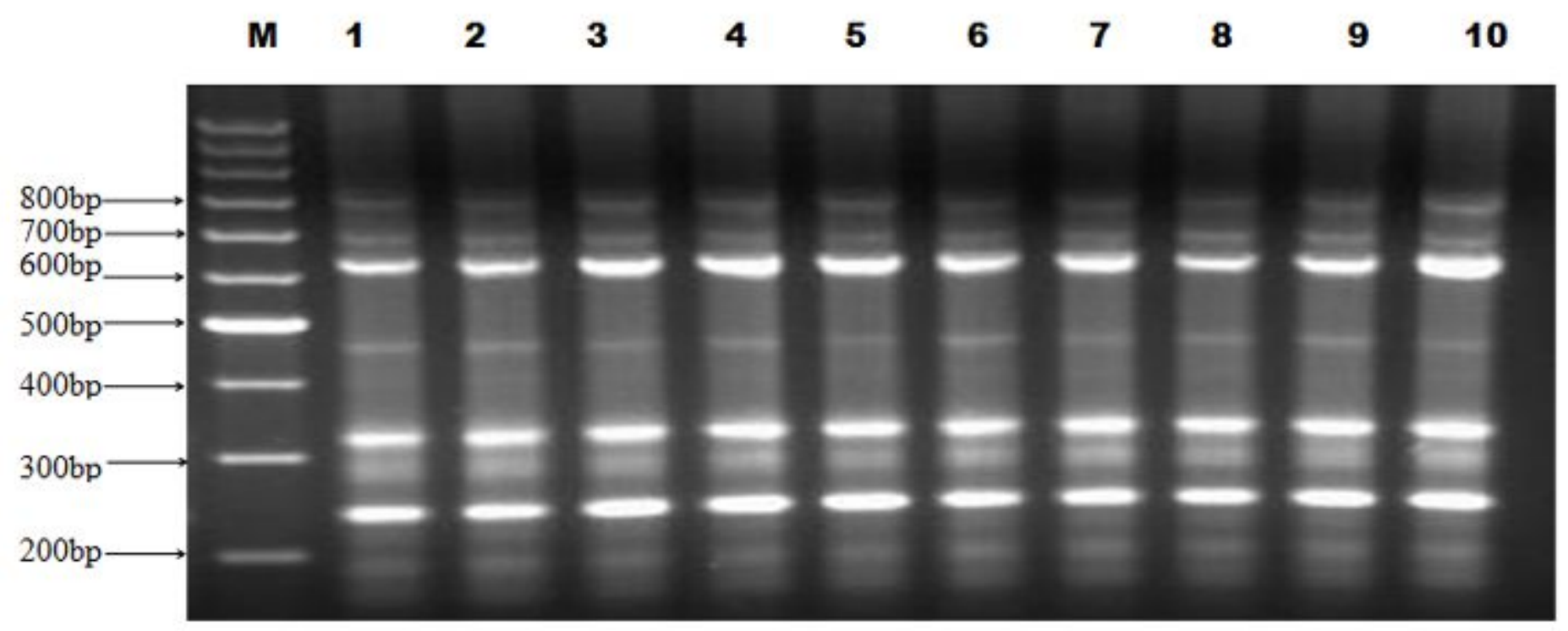

\section{Figure 1}

Representative gel showing banding profiles by ERIC-PCR analysis in ESBLs-producing E. cloacae isolates Footnotes for Figure 1 Note: M: DNA molecular weight; 1 13: ESBLs-producing E. cloacae isolates from different samples in ICU 1-2: strains isolated from pus; 3-4: strains isolated from urine; 5-6: strains isolated from blood; 7: strains isolated from ascite; 8-9: strains isolated from cathers and drainage tube; 11-13: strains isolated from sputum or tracheal secretions; ERIC: Enterobacterial repetitive intergenic consensus 


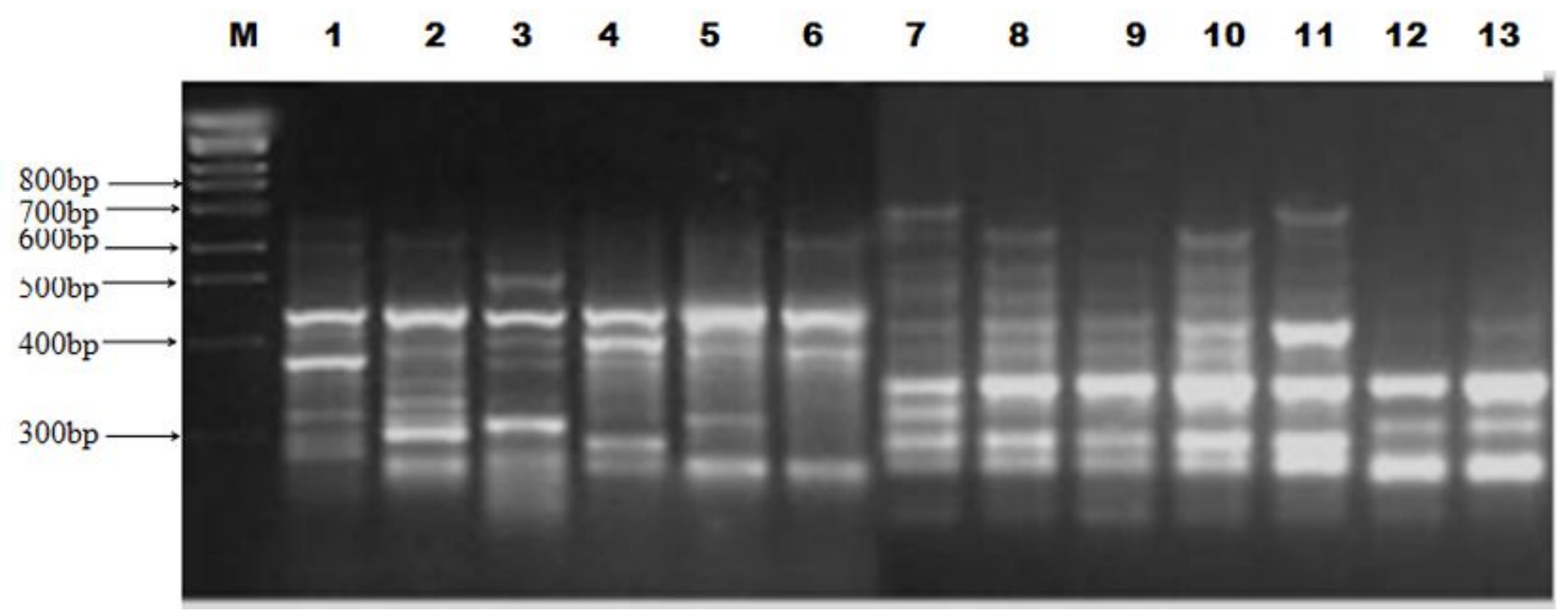

Figure 2

Representative gel showing banding profiles by ERIC-PCR analysis in ESBLs-producing E. coli isolates Footnotes for Figure 2 Note: M: DNA molecular weight; 1 13: ESBLs-producing E. coli isolates from different samples in ICU 1-2: strains isolated from pus; 3-4: strains isolated from urine; 5-6: strains isolated from blood; 7: strains isolated from ascite; 8-9: strains isolated from cathers and drainage tube; 11-13: strains isolated from sputum or tracheal secretions; ERIC: Enterobacterial repetitive intergenic consensus 


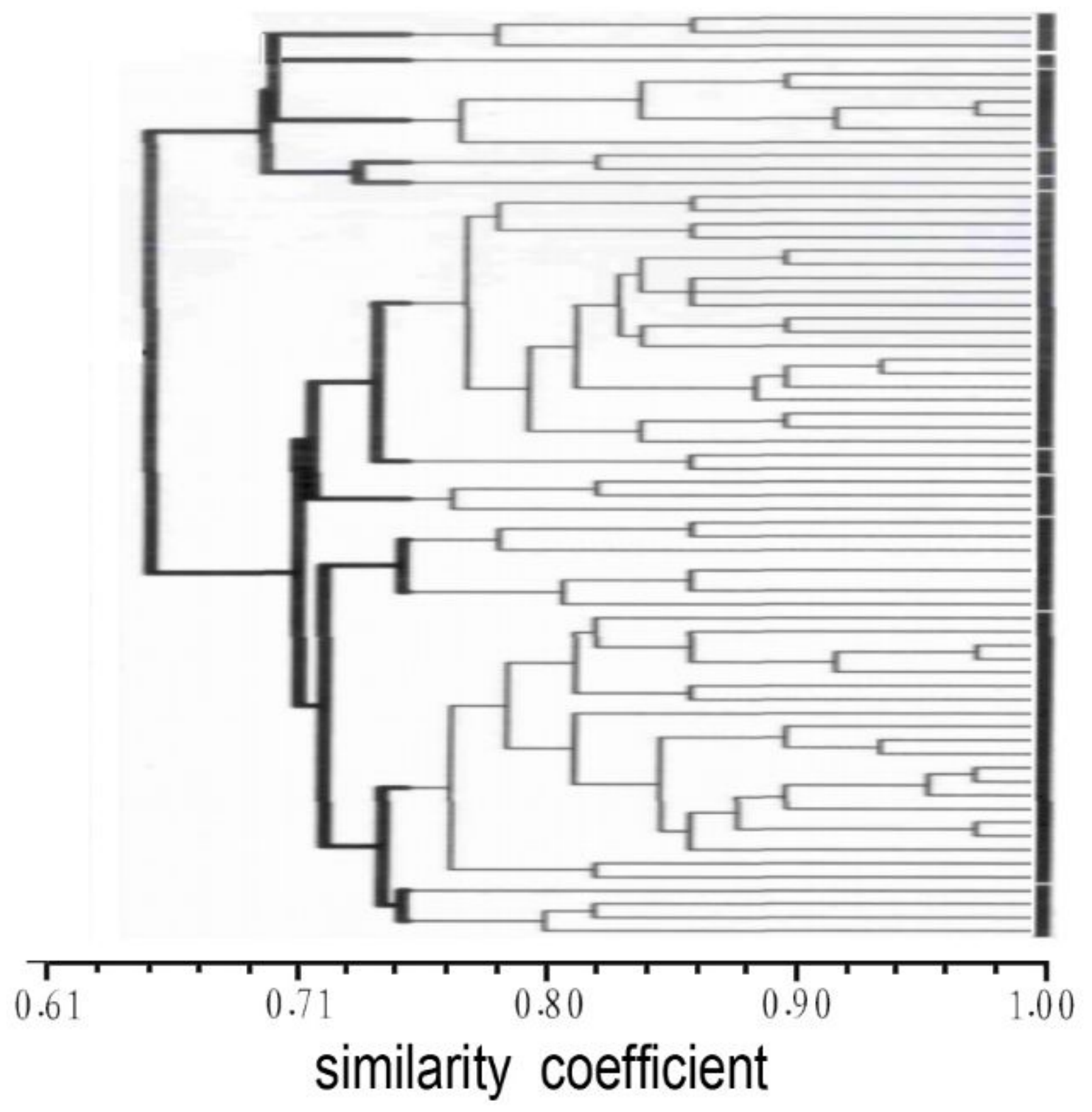

Figure 3

Dendrogram from ERIC-PCR analysis in ESBLs-producing E. coli isolates Footnotes for Figure 3 Note: The scale bar showed the similarity values. ERIC: Enterobacterial repetitive intergenic consensus

\section{Supplementary Files}

This is a list of supplementary files associated with this preprint. Click to download.

- Coverletter.doc 